Interactive comment on "Measuring ocean surface velocities with the KuROS and KaRADOC airborne near-nadir Doppler radars: a multi-scale analysis in preparation of the SKIM mission" by Louis Marié et al.

Ernesto Rodriguez (Referee)

ernesto.rodriguez@jpl.nasa.gov

Received and published: 26 February 2020 


\title{
Evaluation of "Measuring ocean surface velocities with the KuROS and KaRADOC airborne near-nadir Doppler radars: a multi-scale analysis in preparation of the SKIM mission" by Marié et al.
}

\author{
Ernesto Rodriguez
}

February 26, 2020

\section{Summary Evaluation}

The SKIM mission is based on the concept of measuring total surface velocity using near-nadir Doppler scatterometry. One of the critical factors in the feasibility of this concept is demonstrating the ability to remove the velocity signature of gravity waves, which, following previous work by Nouguier et al. (2018), can be 20 to 30 times the value of the Stokes drift. This can result in wave induced signatures on the order of $2 \mathrm{~m} / \mathrm{s}$ to $3 \mathrm{~m} / \mathrm{s}$, which are more than an order of magnitude greater than the desired current accuracy.

The main purpose of this paper is to demonstrate that this is feasible using the current model. To show this, the team has deployed two Doppler scatterometers (at Ku and Ka-bands) together with significant in situ resources, including a buoy to obtain surface 
wave spectra, HF-radar, and two kinds of drifters drogued at different depths. The final results of the paper show a good agreement between the theory of Nouguier et al. at Ka-band (although see detailed comments below), the band proposed for SKIM, but poor agreement at Ku-band and a different frequency dependence between Ka and Ku than predicted by the theory.

The experiment was carefully and thoughtfully designed and the team has made a significant effort to characterize the instruments, especially as regards the mean behavior of the signal. Some discussion has been devoted to the effects of antenna beamwidth at Ku-band leading to contamination of the Doppler signal due to the variation of the radar cross section within the radar footprint. However, given the qualitative discrepancy between theory and observations, additional effort should be devoted to quantifying the measurement errors to show that the Ku-band observations could be compatible with the theory, given feasible measurement uncertainties. Alternately, physical sources for the discrepancy should be identified for future avenues of study. A more detailed suggestion is given below.

Overall, the paper has a logical outline. However integration of the different sections into a consistent style and level of detail has not been as successful, leading to some repetition and confusion, at times. The paper would benefit by a final integration to sharpen the presentation into a more uniform manuscript.

In spite of these reservations, I think that the data collected are an important data set that should be in the open literature and recommend its publication, hopefully after some of the more detailed comments below have been addressed. I recommend that the authors consider putting the data in the public domain, so that it can serve to lay the groundwork for work that will strengthen the case for the SKIM mission. 


\section{Error Quantification}

OSD

Although there is a numerical discussion of various error sources (especially biases due to the antenna pattern and azimuthal variations of backscatter cross section), there is no attempt at deriving an error budget for either of the instruments. This would not be important if the observed measurement scatter were small. However, it is far from small, as can be seen in Figures 12, 13 and 16, where measurement standard deviations varying from $1 \mathrm{~m} / \mathrm{s}$ to $2 \mathrm{~m} / \mathrm{s}$ can be observed. Figure 12 is very enlightening about the variation characteristics of the Ka-band measurements, and an equivalent version would have been very useful for Ku-band. For SKIM, it is important to show that not only the model predicting the mean behavior is understood, but also that the error performance is understood. Currently, this information is not contained in the paper, but all the data are available to produce this validation.

The error budget should contain, at least: 1) Expected measurement random velocity errors, which can be calculated in a straightforward fashion from the pulse pair correlation. 2) Contributions from pointing errors. For KuROS, the incidence angle is very well constrained by the high range resolution (although platform elevation couples in at shallow angles, as noted by the authors), but this is not the case for KaRADOC, where a single footprint is used. Typical aircraft roll (and, to a lesser extent, pitch) variations will lead to variations in the local incidence angle of up to a few degrees (leading to large errors, if uncorrected), and it is not clear in the description of the processing how these effects are mitigated. 3) Error bounds on the possible Doppler effects due to uncertainties in the antenna pattern. 4) Error bounds on the expected effects of the sigma0 azimuth modulation errors as a function of azimuth, which can be obtained using the wavelength of the resolved waves, shown in Figure 9. 5) Modeling assumptions (see below).

Both radar systems have high PRF to properly sampling the Doppler. Is the contamination due to range ambiguities significant? Has it been considered as a source of

Interactive comment 
error?

Examination of Figure 12 shows passes in the east-west direction have lower levels of OSD variations than those going north-south. In addition, the frequency of variation is higher on the 22nd than on the 24th, but the amplitude of variability is larger on the 24th. What is the reason for this? It does not seem to align with wind or wave directions. In any case, the characteristics of the variations seem to be long-wavelength, leading one to suspect either attitude errors or errors due to the changes in the surface field characteristics. Examining the equivalent noise characteristics of the Ku-band data would potentially help in understanding the differences between the two frequencies.

One observation is that, comparing the variations in Figure 16 and 13, the level of within track variability is smaller for Ku band than for Ka-band. Thus the lack of agreement with the model is not due to higher random noise (as could be expected from wave sigma0 contamination), but through some systematic azimuth dependent effect. One potentially useful exercise is to assume that the azimuth brightness gradient contains additional harmonics to the ones estimated in going from Fig. 16a to 16b. Is it possible to account for the divergence from the model with these higher harmonics? If so, are these excluded by the sigma0 observations? Can they be ascribed to systematic coupling that might happen between the antenna pointing and the attitude? If these explanations are not feasible, does this indicate that additional physics needs to be incorporated into the model (at least at Ku-band)?

\section{Modeling and retrieval issues}

There seems to be some mixed messages regarding the modeling assumptions. In Nouguier et al. (2018), a Gaussian assumption is made throughout. On the other hand, the authors quote the asymmetry and skewness of the slope distribution (with references to Munk (2008) and Chapron et al. (2202)) in order to explain the

Interactive comment
Printer-friendly version

Discussion paper 
upwind/downwind asymmetry in the Ku-band backscatter cross-section (Figure 10), which is not insignificant. In equation 16, the isotropic backscatter curves of Nouguier et al. (2016) are used, but they are multiplied by an azimuthal modulation factor $F(\varphi)$, which is not in the original paper and which does not seem to show up again in the analysis. Was such a factor used? If so, is it related to the azimuthal modulation factor quoted in the azimuth modulation fits quoted (but whose values are never given) in the second paragraph in page 21? If not, where is it coming from? Backscatter data are collected at Ku-band and presented in Figure 10A. Do these backscatter data fit the model in equation 16 ? If so, are the azimuthal modulations derived from these data for both $\mathrm{Ku}$ and $\mathrm{Ka}$ ? If not, is there a justification for using equation 16 when it does not match the data?

In the Nouguier et al. (2018) paper, there are two models presented: one for range resolved or not range resolved Dopplers. Since KaRADOC is not resolving the waves, I assume that the second model is used. This model contains two parts (equation 15, Nouguier et al. (2018)), one which dominates along the wave direction, and another one which has contributions at other azimuths. In this paper, only one terms seems to have been kept (i.e., equation 15, Nouguier et al. (2018)). What is the justification for neglecting the second contribution at other azimuths?

It is well known that non-Gaussian effects will lead to a correlation between the modulation of the slope rms and the location along the wave phase. This effect leads to the EM bias in altimetry, for example. Will the level of modulation consistent with EM bias results lead to a change in the predictions made by the model? Will it lead to an upwind-downwind asymmetry in the Doppler? Can it partially account for the 10percent adjustment that had to be made to make the model predictions fit the data?

In the retrieval of the surface currents, it was assumed that the current in the scene remained constant. However, as shown in Table 2 and Figure 7, there was significant change in the currents due to tidal variations measured by the Trefle buoy. How was this accounted for during the fitting? The HF-radar imager linked to in the paper also 
show some current gradients in the region: were the observable by the radars?

Table 2 also shows significant disagreement between the Trefle buoy velocities and

OSD those from the other in situ data. Could you comment on the source of discrepancy?

\section{Miscellaneous comments}

Interactive

comment

Figure 5 appears with insufficient attribution or description. Part of it comes from Nouguier et al. (2016), but there are additional subpanels whose provenance should be clarified.

The term mss $_{\text {shape }}$ is introduced with just a reference to Nouguier et al 2016. To make things easier for the reader, it should be clarified that it is the apparent rms slope obtained by fitting the backscatter curves.

In page 32, there is a statement made about the equivalent depth of the measurements from near-nadir Doppler scatterometry. However, no such derivation is presented in the papers referenced. It would be useful to the community of this statement were backed with a calculation for the two wind speeds (perhaps as an appendix).

Interactive comment on Ocean Sci. Discuss., https://doi.org/10.5194/os-2019-77, 2019. 